Doug Geisler, Eva K. Grebel, and Dante Minniti, eds.

\title{
The HST Census of Nuclear Star Clusters in Late-type Spirals
}

\author{
Torsten Böker \\ Space Telescope Science Institute, 3700 San Martin Drive, Baltimore, \\ MD 21218, U.S.A.
}

\begin{abstract}
I describe preliminary results from an I-band snapshot survey with HST/WFPC2 of nuclear star clusters in late-type spiral galaxies. The goal of the program is to derive the fraction of galaxies that harbor a nuclear star cluster, and to analyse their photometric and structural properties. Together with follow-up spectroscopy, the survey will illuminate the formation mechanism of nuclear star clusters and the implications for the dynamical and morphological evolution of the host galaxy.
\end{abstract}

\section{Introduction}

Over the past decade, high dynamic range observations with modern CCD detectors have shown that compact stellar nuclei are a common feature of spiral galaxies of all Hubble types (e.g. Matthews et al. 1999; Corollo, Stiavelli, \& Mack 1998). In order to answer whether such nuclear star clusters form repeatedly, or only once one must obtain the age distribution of nuclear star clusters. So far, however, reliable age estimates exist for only a handful of objects. Surprisingly, many of the nuclei studied in detail so far appear to be rather young. Examples include the Milky Way ( 3 Myrs, Krabbe et al. 1995), NGC 3227 (50 Myrs, Schinnerer, Eckard, \& Tacconi 2001), as well as M31 and M33 (Lauer et al 1998). More recently, we have published nuclear cluster ages derived from ground-based spectroscopy for IC 342 ( $\leq 60$ Myrs, Böker, van der Marel, \& Vacca), and NGC 4449 (6-10 Myrs, Böker et al. 2001).

However, it is possible that ground-based observations predominantly target the brightest and hence youngest clusters. In order to get a more representative picture of nuclear star clusters, it is important to study a galaxy sample without bias towards the high end of the nuclear cluster luminosity range. We have therefore selected all late-type (Scd or later) spiral galaxies within $v_{z} \leq 2000 \mathrm{~km} / \mathrm{s}$. In order to minimize the effects of extinction due to the galaxy disk, we excluded all objects with high inclination $\left(\mathrm{R}_{25} \equiv \log (\mathrm{a} / \mathrm{b})>0.2\right.$ from the sample. The remaining 113 galaxies were used as the target pool for our WFPC2 snapshot program. All images are taken with the WFPC2 camera onboard HST through the F $814 \mathrm{~W}$ (I-band) filter.

To illustrate the data products we expect to obtain for a large fraction of our snapshot sample, Figure 1 shows the I-band image and SB profile of NGC 4540. For the 14 objects fully analyzed todate, the figure also shows plots of some correlations between cluster and host galaxy properties. The full data 

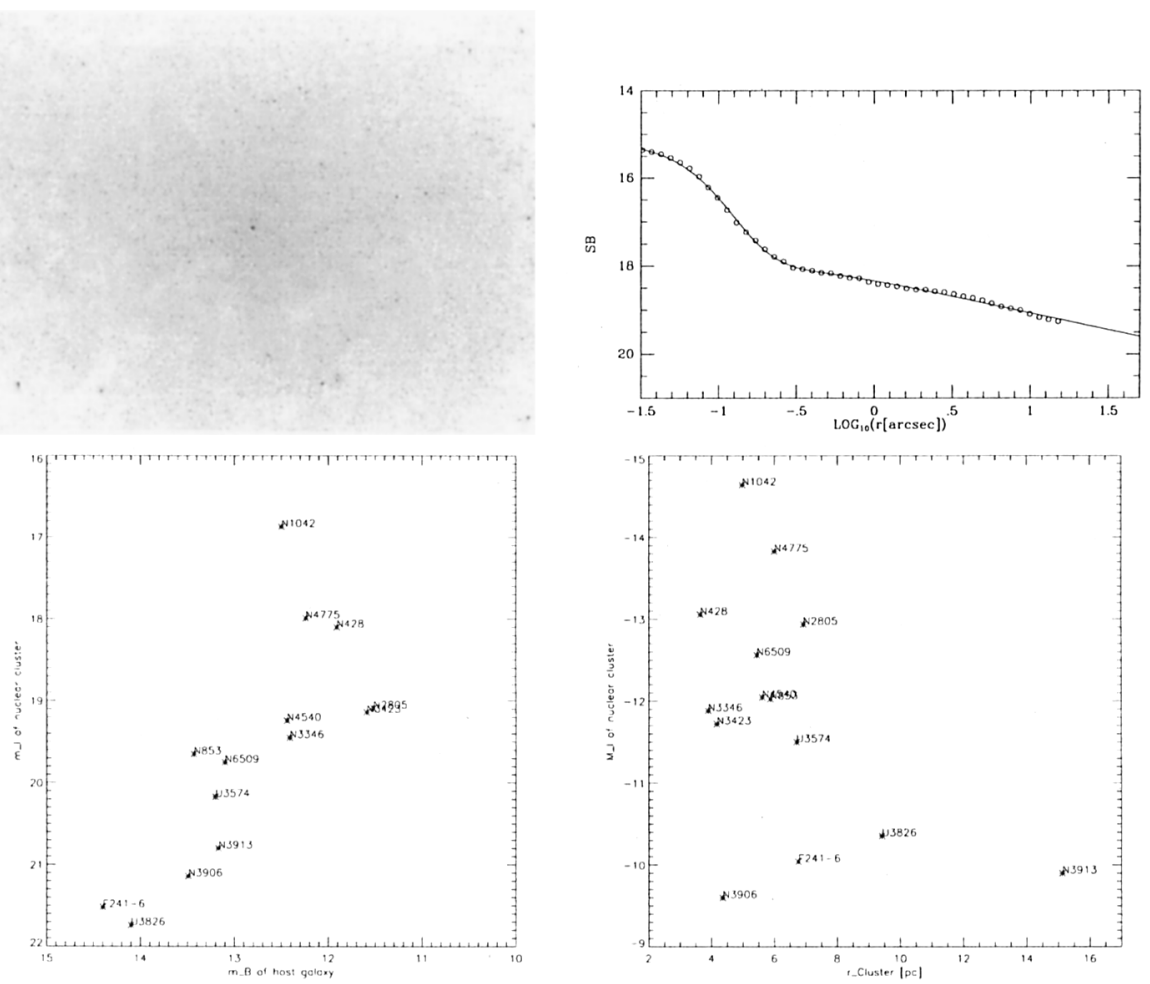

Figure 1. Top: HST WFPC2 I-band image of the central $30^{\prime \prime} \times 20^{\prime \prime}$ of NGC 4540, and SB profile derived from ellipse fitting. The solid line is a generalized Nuker-law fit. Bottom: correlations between $m_{I}$ of the nuclear cluster and $m_{B}$ of the host galaxy (left), and between physical size and absolute luminosity of the nuclear cluster.

set, details of the analysis, and interpretation of the results will be reported in an upcoming paper (Böker et al. 2001, in preparation).

\section{References}

Böker, T., van der Marel, R. P., \& Vacca, W. D. 1999, AJ, 118, 831

Böker, T., et al. 2001, AJ, 121, 1473

Carollo, C. M., Stiavelli, M., \& Mack, J. 1998, AJ, 116, 68

Krabbe, A. et al. 1995, ApJ, 447, L95

Lauer, T. R., et al. 1998, AJ, 116, 2263

Schinnerer, E., Eckard, A., \& Tacconi, L. J. 2001, ApJ, 549, 254 